\title{
Activités
}

18-1 | 2021

Le programme de recherche cours d'action (2)

\section{Introduction au dossier}

\section{Germain Poizat et Julia San Martin}

\section{(2) OpenEdition}

Journals

Édition électronique

URL : http://journals.openedition.org/activites/6091

DOI : 10.4000/activites.6091

ISSN : 1765-2723

\section{Éditeur}

ARPACT - Association Recherches et Pratiques sur les ACTivités

\section{Référence électronique}

Germain Poizat et Julia San Martin, «Introduction au dossier », Activités [En ligne], 18-1 | 2021, mis en ligne le 15 avril 2021, consulté le 17 avril 2021. URL : http://journals.openedition.org/activites/6091 ; DOI : https://doi.org/10.4000/activites.6091

Ce document a été généré automatiquement le 17 avril 2021

\section{(c) $(i)(9)$}

Activités est mis à disposition selon les termes de la licence Creative Commons Attribution - Pas d'Utilisation Commerciale - Pas de Modification 4.0 International. 


\title{
Introduction au dossier
}

\author{
Germain Poizat et Julia San Martin
}

1 Ce dossier sur «Le programme de recherche cours d'action" constitue la seconde partie de la publication conjointe des revues Activités (première partie dans le numéro 17-2/2020) et Laboreal (publication d'une sélection d'articles dans le numéro 16-2/2020). Avec l'objectif de rendre compte des récentes avancées issues des études et recherches inscrites dans ce programme de recherche, les articles constituant la première et deuxième partie de ce dossier invitent le lectorat à réfléchir autour des inventions méthodologiques, des dimensions technologiques, et des dispositifs de formation conçus à partir de l'analyse de l'activité humaine et pour la transformation de l'activité.

2 Francisco de Paula Antunes Lima, Rodrigo Ribeiro, Marcelle La Guardia et Samira Nagem décrivent une étude de cas portant sur l'automatisation de petites centrales hydro-électriques qui rend compte des apports du cadre théoriques et méthodologiques du cours d'action pour la prise en compte des éléments sociaux, subjectifs et techniques dans des projets sociotechniques ou anthropocentriques. Ils témoignent du besoin d'une praxéologie empirique pour traiter les interfaces HommeHomme (enjeux de formation) et Homme-Machine (enjeux d'automatisation) des systèmes H-H-M.

3 L'article de Yvon Haradji décrit les apports de la dimension technologique du programme de recherche cours d'action et illustre les tenants d'une recherche technologique à travers une analyse longitudinale du développement d'une plateforme de simulation multi-agents (SMACH) visant à anticiper les comportements humains dans une perspective de réduction de la consommation d'énergie à différents niveaux (habitat, quartier, ville...). Les éléments d'analyse proposés par l'auteur permettent de réfléchir sur la relation organique entre activité et technique, sur les apports de l'analyse de l'activité à différentes étapes de la conception, sur les modélisations en ergonomie, ainsi que sur les critères de validation du programme de recherche technologique.

4 Simon Flandin, Deli Salini, Artemis Drakos et Germain Poizat nous proposent de prolonger la réflexion sur les apports du programme de recherche technologique cours 
d'action en se centrant sur la conception de situations de formation. En considérant l'analyse de formations sur la gestion et le dépassement des événements inédites ou critiques, les auteurs proposent de concevoir les situations de formations comme des occasions de perturbations et/ou relance de la dynamique de significations des acteurs. L'article présente une réflexion sur la notion d'activité-signe et l'intégration des dimensions fictionnelle et événementielle qui permettent de comprendre les dimensions transformatives de ces formations ainsi que leurs effets, et de formuler des principes transversaux de conception.

5 La contribution de Serge Leblanc, Hélène Bouchot et Mélanie Secheppet propose de s'intéresser aux processus d'apprentissage-développement en analysant trois situations de formations basées à des degrés divers sur l'utilisation de la vidéo-formation, la simulation, et l'observation des pairs. Les auteurs présentent une modélisation théorique visant à rendre compte de l'expérience mimétique émergeant de l'observation par des formés des situations vécues par d'autres acteurs (alloconfrontation). Cette modélisation, basée sur l'hexatomie de l'unité du cours d'expérience, rend compte de l'intérêt de ce raffinement méthodologique pour la description des «transformations silencieuses » de l'activité ainsi que des conditions de performativité de l'action.

6 Nous saisissons l'occasion de cette introduction pour rompre avec le style académique traditionnel et présenter dans la continuité de la méthode élémentaire, de la méthode développée, et de la méthode réfléchie du programme de recherche cours d'action, un nouvel essai de méthode : la méthode « illustrée ».

7 Pour certains anthropologues, l'Homme se caractérise non seulement par ses capacités d'action, d'invention, de transmission, ou de réflexion, mais également par ses capacités de relâchement, de distraction, d'absurde, de dérision, et de jeu qui introduisent une « suspension » dans l'action et peuvent donner l'impression d'inutilité ou de gaspillage (e.g., Huizenga, 1951; Le Breton, 2018). Nous considérons pour notre part que le rire se constitue comme un catalyseur des transformations de l'individu. Plusieurs études conduites dans le cadre de programme de recherche cours d'action ont pointé le fait que l'activité se transforme lorsqu'elle présente une dimension fictionnelle (e.g., Salini \& Durand, 2020 ; Salini \& Poizat, 2021 ; Poizat, Salini, \& Durand, 2013), c'est-à-dire une complexité particulière qui la distingue d'un engagement exclusivement pragmatique, direct, sérieux et utilitaire. Autorisons-nous ce débrayage temporaire et salutaire arrivant au terme de ce double dossier autour du programme de recherche cours d'action.

8 Les premières esquisses de cette "méthode illustrée ", présentées ici et en avantpremière, sont nées sous l'impulsion de Jean-Baptiste Haué et de Cécile Barbier en collaboration avec l'illustrateur Thierry Dispan de Floran. Cette Bande Dessinée a été réalisée avec l'aimable collaboration du «service Expérience Utilisateur du groupe Renault ». Le propos ici consiste à faire les choses avec sérieux, mais sans trop se prendre au sérieux ; à exposer la recherche, les hypothèses, et les concepts à travers une mise en images par la bande dessinée; et à exploiter les dimensions iconiques et métaphoriques de l'écriture en bande dessinée pour favoriser les pontages et l'appropriation de ces hypothèses et concepts.

9 Les lecteurs assidus ou plus ponctuels des études conduites dans le cadre du programme cours d'action savent que ces textes utilisent un vocabulaire et un positionnement théorique qui revendiquent une certaine spécificité (rendant parfois 
les écrits particulièrement denses pour ne pas dire énigmatiques). Or quand il faut rendre accessibles des éléments nouveaux et/ou complexes (expériences, concepts, construits théoriques), les métaphores sont des offres de signification permettant la compréhension "globale » de ce qui est perçu préalablement comme vague et difficile, par un repli sur l'essentiel. Le mode de signification métaphorique présente deux avantages. Il fait l'économie (sémiotique ou cognitive) d'une symbolisation conventionnelle et formelle, et demeure proche de l'expérience vécue. Et il permet l'identification de régularités et de familiarités basée sur des ressemblances, réduisant ainsi la complexité par des pontages iconiques avec du déjà connu. Malgré ses possibles limites, cette inscription métaphorique est efficiente : elle fait « sentir les choses » tout en créant les conditions d'une symbolisation abstraite et formelle dans un second temps. La métaphore est une modalité de signification particulièrement riche car elle est au cœur de l'imagination, et à la base de la création et de l'invention. L'écriture en bande dessinée cumule ces différents avantages que ce soit pour l'activité de recherche (inventivité conceptuelle, travail interprétatif) ou pour l'appropriation des hypothèses, concepts, et notions par d'autres.

BD Cours d'Action illustré : Une littérature riche

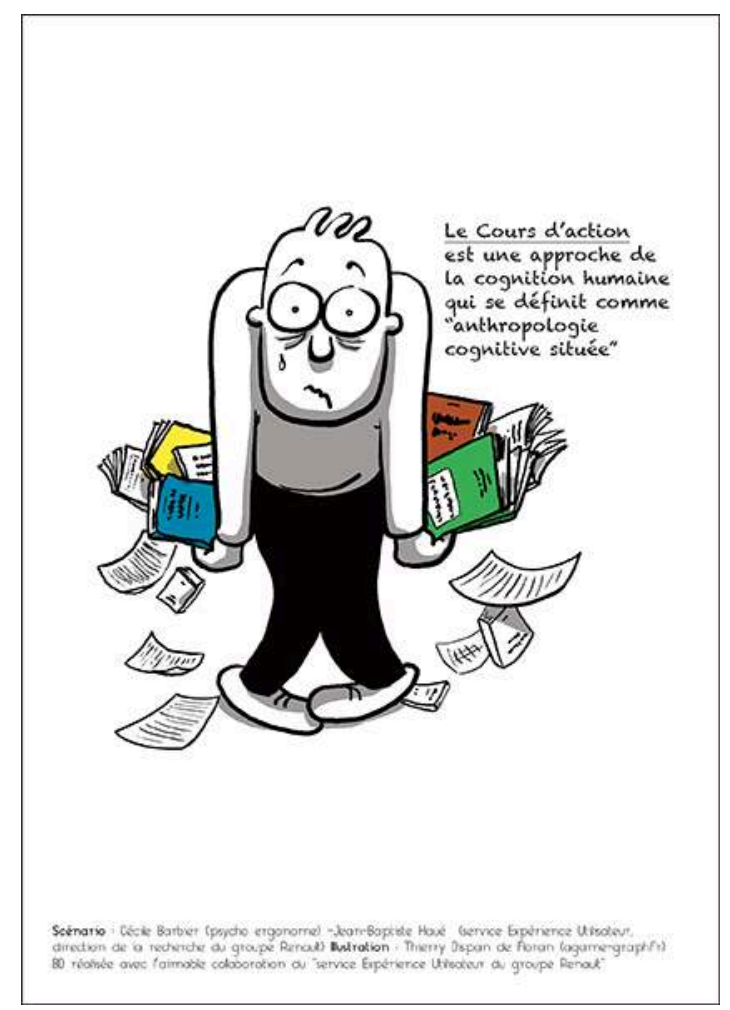

Scénario : Cecile Barbier (psycho-ergonome) - Jean-Baptiste Houé (service Expérience Utilisateur, direction de la recherche du groupe Renault).

Illustration: Thierry Dispan de Floran (https://www.agame-graph.fr).

BD réalisée avec l'aimable collaboration de "service Expérience Utilisateur du groupe Renault".

10 La première planche ci-dessous s'attaque à deux notions centrales dans le cadre du programme de recherche cours d'action (Poizat \& San Martin, 2020): le « couplage structurel » et l'« engagement ». Ajoutons-y celle de « monde propre». Ces notions ont des conséquences ontologiques, épistémologiques, et technologiques dans le cadre du programme de recherche cours d'action. Cette première planche montre comment ces 
notions font écho à une réalité très concrète de nos vies pour ensuite esquisser une présentation du signe hexadique et de ses composantes. On y retrouve Jules et Jim déjà rencontrés dans Durand (2017).

\section{Du couplage asymétrique à la notion d'engagement}

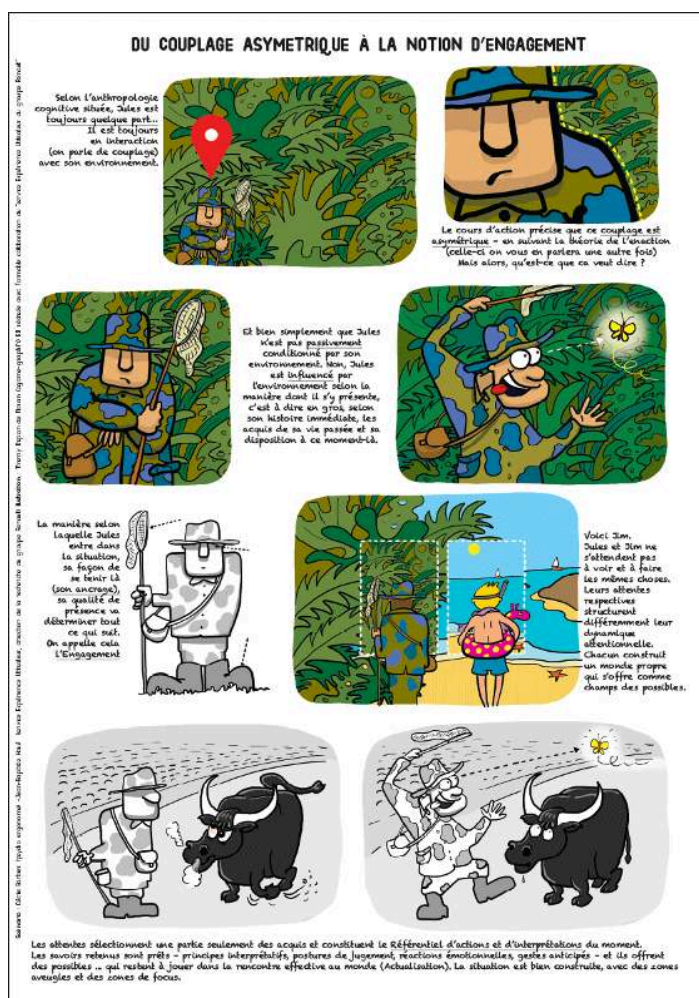

Scénario : Cecile Barbier (psycho-ergonome) - Jean-Baptiste Houé (service Expérience Utilisateur, direction de la recherche du groupe Renault).

Illustration : Thierry Dispan de Floran (https://www.agame-graph.fr).

$\mathrm{BD}$ réalisée avec l'aimable collaboration de "service Expérience Utilisateur du groupe Renault".

11 La seconde planche présentée ici est une synthèse graphique de l'étude de (Haué, Le Bellu, \& Barbier, 2020) publiée dans la revue Activités et portant sur les dynamiques de désengagement et réengagement dans la conduite du véhicule autonome. Elle illustre l'ingénierie des situations développée dans la cadre de programme de recherche cours d'action et ses dimensions pratiques. Elle traite notamment des impacts de la conception d'un objet technique sur l'activité de conduite et les recommandations associées formulées par les ergonomes sous une forme visant à faciliter l'appropriation au sein de l'entreprise et du collectif de conception des résultats et recommandations produits. 


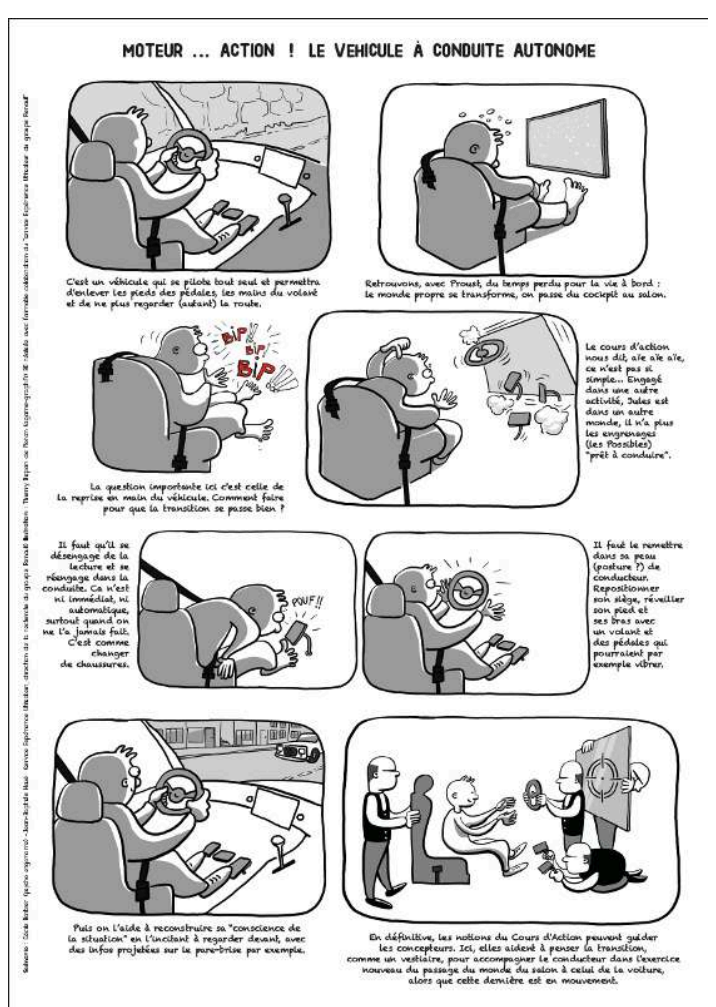

Scénario : Cecile Barbier (psycho-ergonome) - Jean-Baptiste Houé (service Expérience Utilisateur, direction de la recherche du groupe Renault).

Illustration : Thierry Dispan de Floran (https://www.agame-graph.fr).

BD réalisée avec l'aimable collaboration de "service Expérience Utilisateur du groupe Renault".

Ces planches sont également une bonne occasion pour se questionner sur les utilisations possibles de la bande dessinée en lien avec l'analyse de l'activité. L'écriture en bande dessinée peut être convoquée pour dévoiler l'activité réelle du chercheur que ce soit lors du recueil, de l'analyse ou de la restitution des données. Plusieurs bandes dessinées, produites dans le champ des sciences sociales, illustrent les épreuves et les routines du travail scientifique. L'émergence de la bande dessinée documentaire ou de la bande dessinée « du réel » ouvre également de nouvelles voies pour tout chercheur obsédé (à juste titre) par le fait de rendre compte du travail réel des acteurs. Elle devient alors une modalité de double-exposition ou de re-présentation de l'activité réelle. Enfin, l'écriture en bande dessinée peut faire partie intégrante des méthodologies de recueil de données, et particulièrement au travail ethnographique, dans le cadre de l'analyse de l'activité. Autrement dit, la création d'une bande dessinée peut être mobilisée à différentes étapes de la démarche de recherche, et probablement de manière encore plus féconde et inventive si des hypothèses épistémologiques et de conception accompagnent cette intégration.

Reprenons ici à notre compte la notion de "pop'philosophie ", chère à Deleuze et popularisée par l'Anti-CEdipe (Deleuze \& Guattari, 1972), en caressant le rêve d'une "pop'méthode». À l'image de la philosophie, il s'agirait d'un rêve, celui d'un programme cours d'action ou d'une analyse de l'activité qui pourrait toucher immédiatement un public non spécialiste et essaimer à la manière de la "culture pop " et du " pop art », avec des adhésions collectives à la fois spectaculaires et spontanées (comme par exemple Woodstock et la musique rock). La proposition formulée ici est 
bien plus transgressive qu'un simple passage d'un objet populaire - films, séries télé, romans, morceau de musique - au crible de la philosophie ou de la recherche académique. Une pop'méthode impose, comme le dit Deleuze, une méthode et une lecture " en intensité », et une rupture avec la rationalité sobre, froide, éteinte du style universitaire. «Pop est le bruit que fait la boite lorsque le couvercle saute» (de Sutter, 2019, p. 27).

\section{BIBLIOGRAPHIE}

Deleuze, G., \& Guattari, F. (1972). L’Anti-CEdipe: Capitalisme et schizophrénie 1. Paris : Les Éditions de Minuit.

Durand, M. (2017). L'activité en transformation. In J.-M. Barbier \& M. Durand (Eds.), Encyclopédie d'analyse des activités (pp. 33-55). Paris : PUF.

Haué, J-B, Le Bellu, S., \& Barbier, C. (2020). Le véhicule autonome : se désengager et se réengager dans la conduite. Activités, 17(1), https://doi.org/10.4000/activites.4987

Huizenga, J. (1951). Homo ludens. Paris : Gallimard.

Le Breton, D. (2018). Rire : Une anthropologie du rieur. Paris : Éditions Métailié.

Poizat, G., Salini, D., \& Durand, M. (2013). Approche énactive de l'activité humaine, simplexité, et conception de formations professionnelles. Education Sciences \& Society, 4, 97-112.

Poizat, G., \& San Martin, J. (2020). Le programme de recherche « cours d'action » : repères historiques et conceptuels. Activités, 17(2), https://doi.org/10.4000/activites.5277

Salini, D., \& Durand, M. (2020). Overcoming a lived experience of personal impasse by creating a theatrical drama: An example of promoting resilience in adult education. In L. McKay, G. Barton, S. Garvis, \& V. Sappa (Eds.), Arts-based research, resilience and well-being across the lifespan (pp. 169-189). Palgrave Macmillan : Cham.

Salini, D. \& Poizat, G. (2021). Dénouements possibles de l'expérience d'impasse : pistes de compréhension et perspectives développementales. L'Orientation scolaire et professionnelle, 50, 61-84.

Sutter (de), L. (2019). Qu'est-ce que la pop'philosophie ? Paris : PUF.

\section{AUTEURS}

\section{GERMAIN POIZAT}

Université de Genève, Suisse - germain.poizat@unige.ch

JULIA SAN MARTIN

Universidad de Aysén, Chile - julia.sanmartin@uaysen.cl 